\title{
Obstructive ileus caused by phlebosclerotic colitis
}

\author{
Seung Hyun Lee, Jong Wook Kim, Se Jin Park, Ju Yeol Heo, Woo Hyun Paik, Won Ki Bae, Nam-Hoon Kim, \\ Kyung-Ah Kim, June Sung Lee \\ Department of Internal Medicine, Inje University Ilsan Paik Hospital, Goyang, Korea
}

A 57-year-old man with chronic kidney disease and a history of using numerous herbal medications visited Inje University Ilsan Paik Hospital for abdominal pain and vomiting. An abdominal radiograph showed diffuse small bowel distension containing multiple air-fluid levels and extensive calcifications along the colon. Computed tomography showed colon wall thickening with diffuse calcification along the colonic mesenteric vein and colonic wall. Colonoscopy, performed without bowel preparation, showed bluish edematous mucosa from the transverse to the distal sigmoid colon, with multiple scar changes. At the mid transverse colon, a stricture was noted and the scope could not pass through. A biopsy of the stricture site revealed nonspecific changes. The patient was diagnosed with phlebosclerotic colitis. After the colonoscopy, the obstructive ileus spontaneously resolved, and the patient was discharged without an operation. Currently, after 2 months of follow-up, the patient has remained asymptomatic. Herein, we report the rare case of an obstructive ileus caused by phlebosclerotic colitis with a colon stricture.

(Intest Res 2016;14:369-374)

Key Words: Phlebosclerotic colitis; Phlebosclerosis; Herbal medicine; Ileus

\section{INTRODUCTION}

Phlebosclerotic colitis (PC) is a rare disease; chronic mesenteric ischemia that is characterized by calcification of the mesenteric vein and thickening of the colonic wall, which result in ischemic colitis. ${ }^{1}$ Ischemic colitis is primarily caused by arterial obstruction secondary to arteriosclerosis, thrombosis, or embolism in the left-sided colon. However, PC is affected by fibrosis and sclerosis of the mesenteric vein that cause colonic congestion. ${ }^{1,2}$ The underlying pathophysiology is unclear, but a few studies have suggested that it might be related to toxins, calcinosis, Raynaud phenomenon, esophageal dysmotility, sclerodactyly, and telangiectasia (CREST) syndrome, Churg-Strauss syndrome, lymphocytic phlebitis, portal hypertension, cardiac disease, diabetes mellitus,

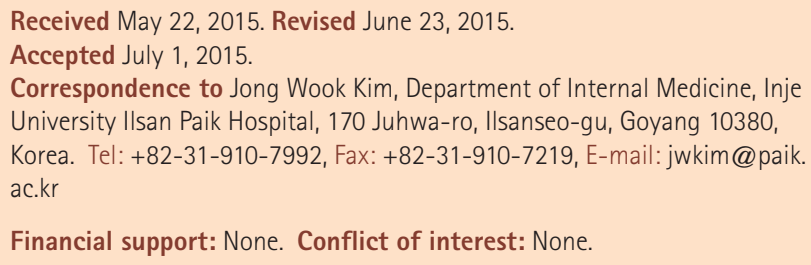

Financial support: None. Conflict of interest: None.

dyslipidemia, or hemodialysis. ${ }^{3,4}$ PC usually involves the proximal colon and has no obvious cause, but certain toxins absorbed from the right-sided colon have been implicated in causing chronic venous damage. ${ }^{2}$ We report a case of PC with a transverse colonic stricture in a patient with chronic kidney disease, who had been taking multiple kinds of herbal medications for over 6 months.

\section{CASE REPORT}

A 57-year-old man visited Inje University Ilsan Paik Hospital after experiencing abdominal pain, nausea, and vomiting for 3 weeks. He had visited our emergency room for the same symptoms 2 weeks prior, where he was suspected of having paralytic ileus. However, he was discharged against our medical advice, insisting that his symptoms were not severe. His symptoms had not improved since this visit. He had chronic renal disease for 10 years. Instead of taking prescription drugs, he had been using self-manufactured herbal medication for over 6 months. The herbal medication included more than 10 herbal ingredients such as sansisi

\footnotetext{
๑ Copyright 2016. Korean Association for the Study of Intestinal Diseases. All rights reserved.

This is an Open Access article distributed under the terms of the Creative Commons Attribution Non-Commercial License (http://creativecommons.org/licenses/by-nc/4.0)

which permits unrestricted non-commercial use, distribution, and reproduction in any medium, provided the original work is properly cited.
} 
(Gardenia jasminoides), hua shi (magnesium silicate), long gu (Mastodi ossis fossilia or talc), and mu li (Ostrea gigas). He had been taking no other medication except for the herbal medicine. He was a 15-pack-year current smoker, and did not drink alcohol.

The patient looked acutely ill; his blood pressure was $132 / 71 \mathrm{mmHg}$, pulse rate $97 / \mathrm{min}$, respiratory rate $20 / \mathrm{min}$, and body temperature $36.2^{\circ} \mathrm{C}$. The patient's abdomen was distended and tympanic, and showed tenderness without rebound tenderness. In addition, his bowel sounds were decreased. Laboratory tests were as follows: white blood cell $6,970 / \mathrm{mm}^{3}$, hemoglobin $8.1 \mathrm{~g} / \mathrm{dL}$, platelet count 372,000/ $\mathrm{mm}^{3}$, BUN $43 \mathrm{mg} / \mathrm{dL}$, creatinine $4.52 \mathrm{mg} / \mathrm{dL}$, and CRP 0.3 $\mathrm{mg} / \mathrm{dL}$. The electrolyte levels were within normal limits.

A plain radiograph showed diffuse small bowel distension and colon wall calcification along the colonic area (Fig. 1A and B). Because of his reduced renal function, a non-contrastenhanced CT scan was taken, which showed diffuse fluidfilled distension of the small bowel with colonic wall thickening and calcifications in the colon wall and at the mesocolon, suggesting atherosclerotic changes (Fig. 2). Paralytic ileus was suspected, and the patient was initially managed with supportive care including hydration, bowel rest, and nasogastric drainage. However, a follow-up CT taken on hospital day 7 did not show any improvement in bowel distention. Colonoscopy without bowel preparation performed on hospital day 8 showed diffuse bluish edematous mucosal change, multiple scarring, and loss of vascularity from the distal sigmoid to the transverse colon. There was a pinpoint stricture in the transverse colon, $65 \mathrm{~cm}$ from the anus, and scope passage was not
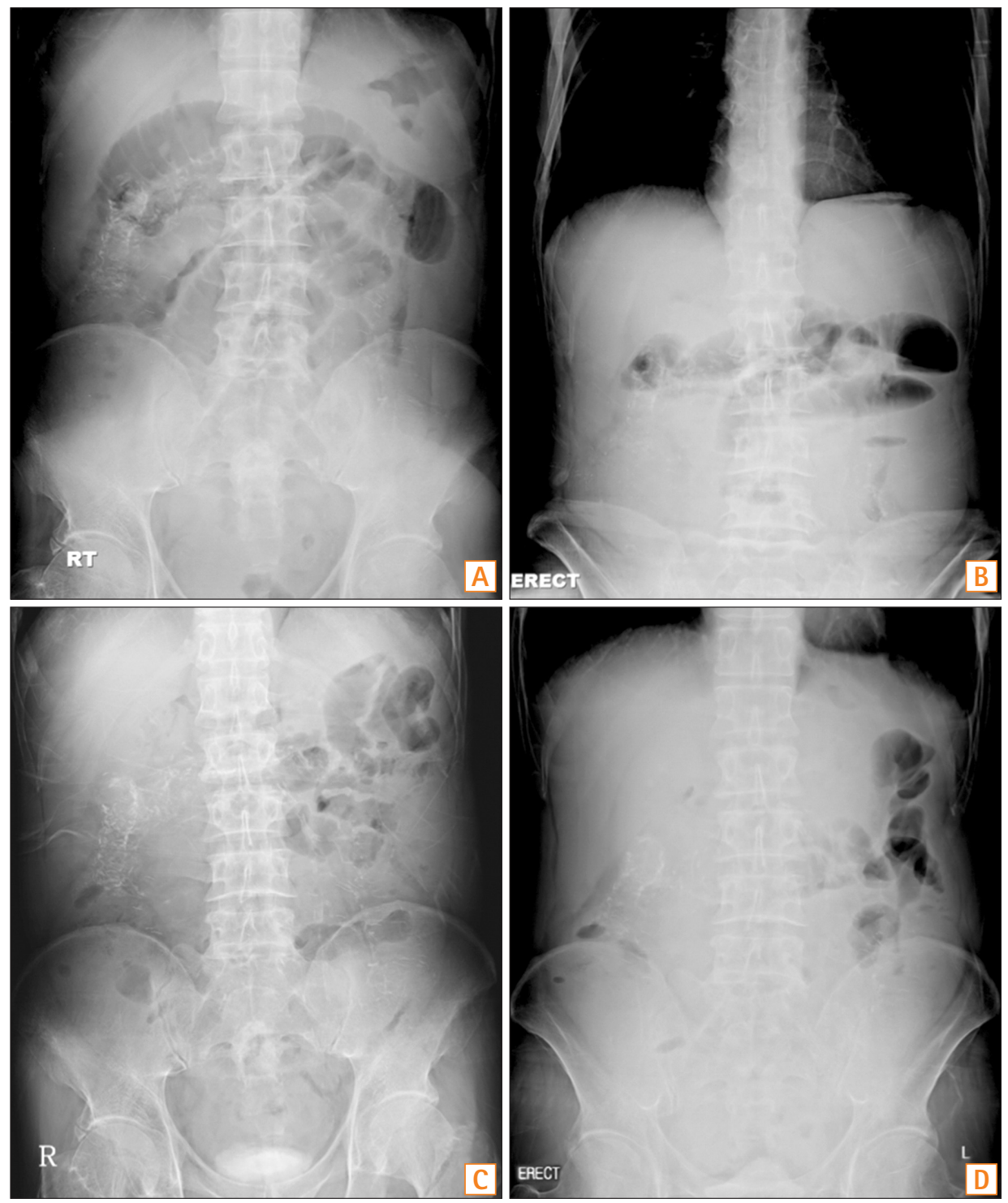

Fig. 1. Plain abdominal radiograph. Initial plain abdominal radiograph shows a diffuse small bowel ileus with multiple airfluid levels and numerous calcification densities along the colon. (A) Supine, (B) Erect. Dramatically improved ileus, 1 day after the colonoscopy. (C) Supine, (D) Erect. 

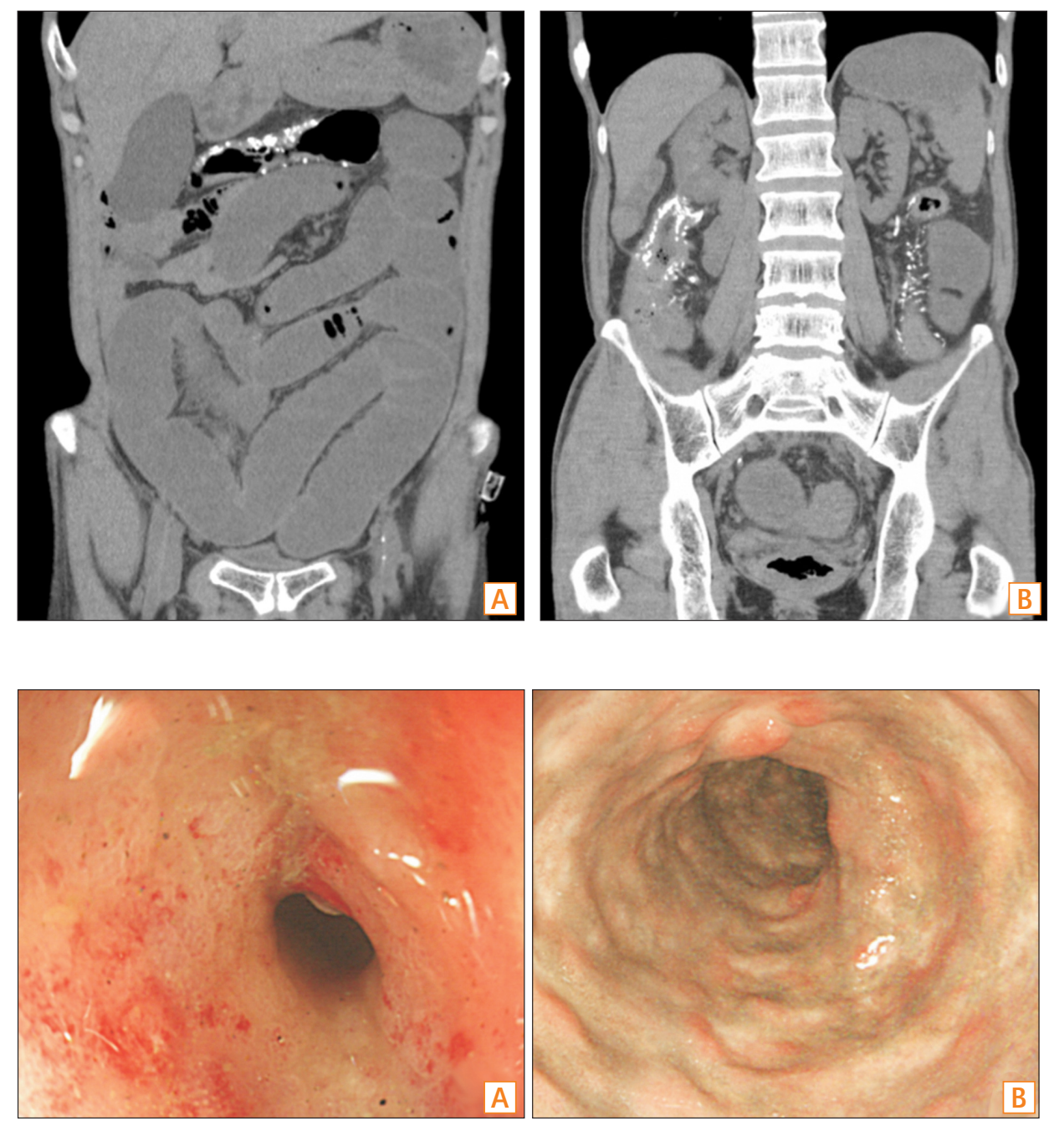

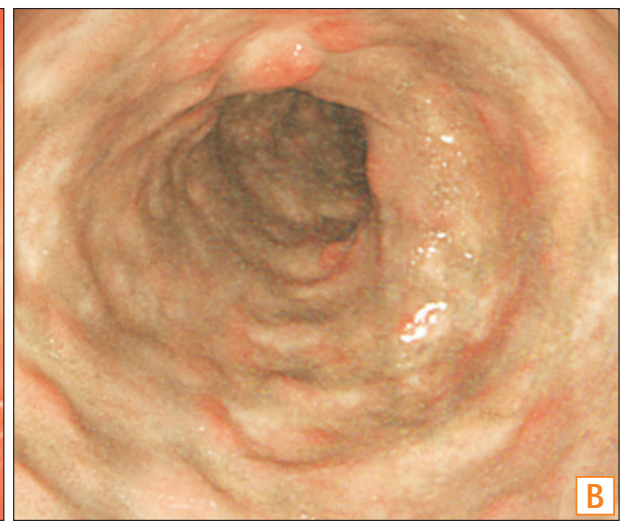

Fig. 2. Non-contrast-enhanced abdominal CT scan. (A) Diffuse fluid-filled distension of small bowel. (B) Colonic wall calcifications.

Fig. 3. Colonoscopy findings. (A) Pinpoint stricture in the transverse colon, scope passage was not possible. (B) Blue-grayish congestive change with diffuse scarring found in the transverse colon.

possible (Fig. 3). A biopsy taken from the stricture site showed chronic inflammation and fibrosis with mild regenerative change (Fig. 4). Under the supposition of a PC diagnosis, a contrast-enhanced CT scan with delayed phase angiography was performed, which showed extensive calcifications along the colonic wall, mesenteric, and portal veins (Fig. 5). Total colectomy was recommended, but the patient refused. Fortunately, on the day after the colonoscopy, the patient's symptoms dramatically improved, and the ileus disappeared (Fig. 1C and D). Presumably, this was due to the dilatational effect from the scope passage attempt, or dislodgement of a fecalith. Based on radiologic, endoscopic, and histologic findings, we diagnosed the patient with PC. After several days of a soft diet, the patient remained asymptomatic, and was discharged. The patient is currently symptom-free, after 2 months of follow-up.

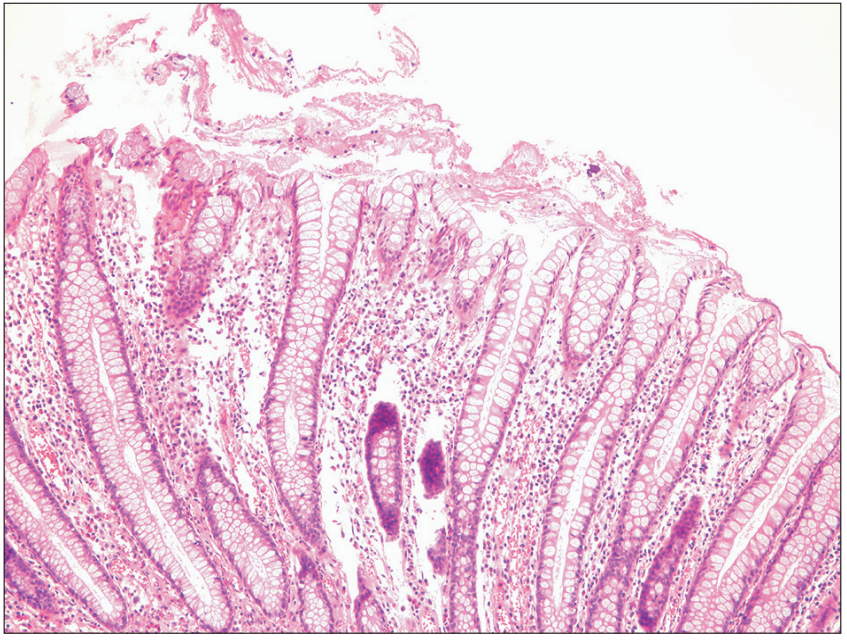

Fig. 4. Pathologic findings at the stricture site. The mucosa shows lymphoid cell infiltration and mild architectural distortion of crypts, which are suggestive of regenerative changes $(\mathrm{HEE}, \times 100)$. No submucosal vessel was included in the specimen. 

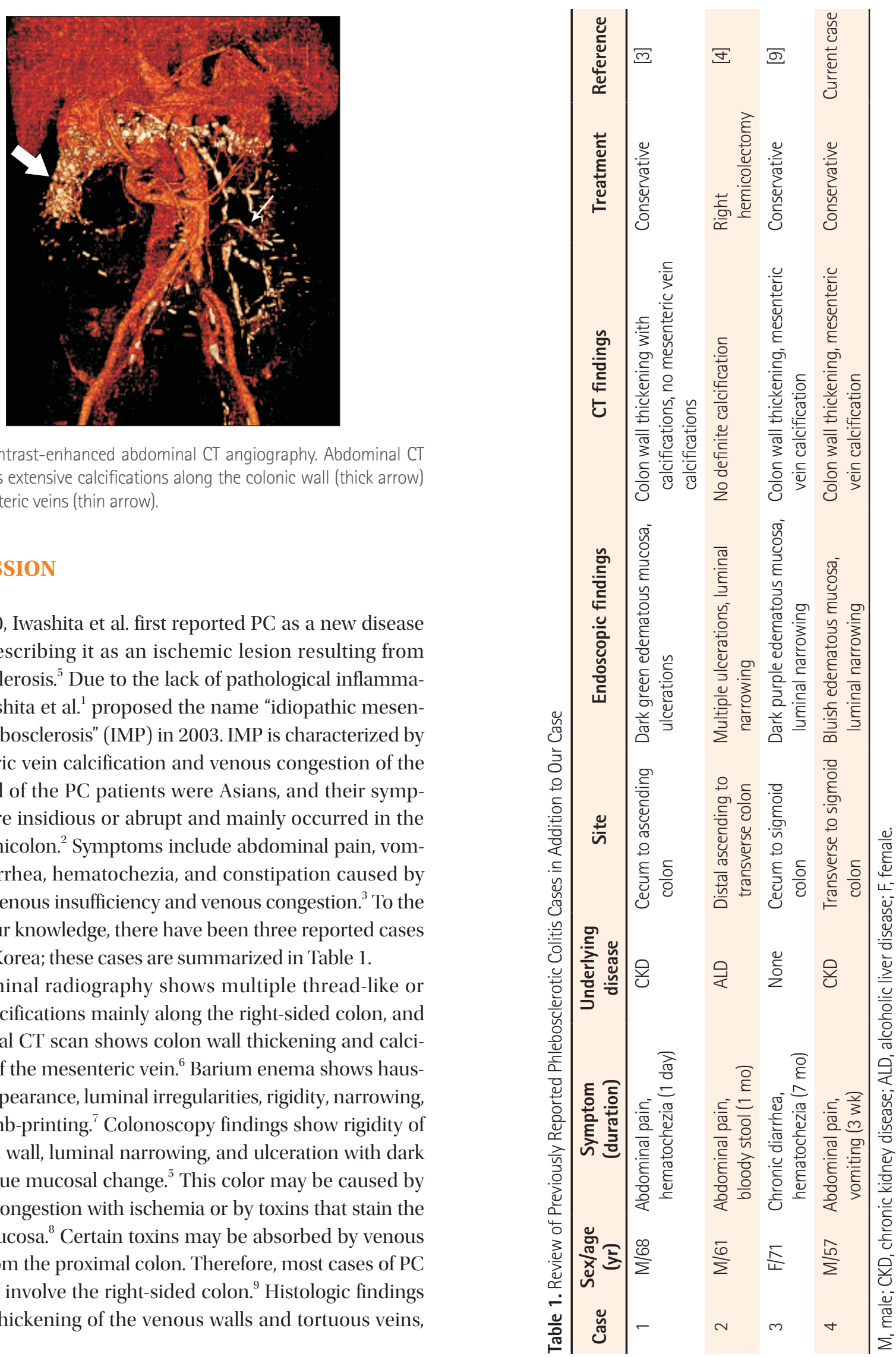

Fig. 5. Contrast-enhanced abdominal CT angiography. Abdominal CT scan shows extensive calcifications along the colonic wall (thick arrow) and mesenteric veins (thin arrow).

\section{DISCUSSION}

In 2000, Iwashita et al. first reported PC as a new disease entity, describing it as an ischemic lesion resulting from phlebosclerosis. ${ }^{5}$ Due to the lack of pathological inflammation, Iwashita et al. ${ }^{1}$ proposed the name "idiopathic mesenteric phlebosclerosis" (IMP) in 2003. IMP is characterized by mesenteric vein calcification and venous congestion of the colon. All of the PC patients were Asians, and their symptoms were insidious or abrupt and mainly occurred in the right hemicolon. ${ }^{2}$ Symptoms include abdominal pain, vomiting, diarrhea, hematochezia, and constipation caused by chronic venous insufficiency and venous congestion. ${ }^{3}$ To the best of our knowledge, there have been three reported cases of PC in Korea; these cases are summarized in Table 1.

Abdominal radiography shows multiple thread-like or linear calcifications mainly along the right-sided colon, and abdominal CT scan shows colon wall thickening and calcification of the mesenteric vein. ${ }^{6}$ Barium enema shows haustral disappearance, luminal irregularities, rigidity, narrowing, and thumb-printing. ${ }^{7}$ Colonoscopy findings show rigidity of the colon wall, luminal narrowing, and ulceration with dark purple-blue mucosal change. ${ }^{5}$ This color may be caused by chronic congestion with ischemia or by toxins that stain the bowel mucosa. ${ }^{8}$ Certain toxins may be absorbed by venous return from the proximal colon. Therefore, most cases of PC primarily involve the right-sided colon. ${ }^{9}$ Histologic findings include thickening of the venous walls and tortuous veins, 
fibrotic degeneration of the venous walls with calcifications, and markedly fibrous thickening in the submucosa of the colonic wall without hemorrhage or thrombosis of the mesenteric vein. ${ }^{2}$ However, it has been suggested that PC may have a relatively long subclinical stage, and calcification within the colonic wall and mesenteric veins at certain stages may not be seen. ${ }^{6}$ In our case, radiologic findings showed mesenteric vein calcifications, but endoscopic biopsy revealed no mesenteric vein calcifications. Since a mucosal biopsy may not be sufficient for evaluation of submucosal vessels, we thought that it was prudent to take only a small amount of tissue, since we anticipated that delayed wound healing by venous congestion and biopsy-induced bleeding could be problematic.

The underlying pathophysiology of PC is not well known, but dialysis, portal hypertension, diabetes, and vasculitis have been suggested as possible causes., Many studies report that PC is related to herbal medicine. Recently, after reviewing 25 cases of $\mathrm{PC}$, Hiramatsu et $\mathrm{al}^{8}{ }^{8}$ reported that a commonly used herbal ingredient, sansisi (gardenia fruit), was correlated with PC. Our patient also had a history of longterm herbal medication usage, including sansisi (gardenia fruit). However, our patient had also been taking many other kinds of herbal ingredients, including zhi zi ( $G$. jasminoides), hua shi (magnesium silicate), long gu (Mastodi ossis fossilia or talc), and mu li (Ostrea gigas), so it is not clear which ingredient caused PC. Another study reported that stopping herbal medicines improved the patient's symptoms. ${ }^{10}$ However, although our patient was strongly advised not to take the herbal medicines, he persistently ingested them, even during the hospital admission, due to his firm belief in the efficacy of the herbal medication.

The management of PC ranges from supportive care to surgery, based on disease severity. PC may be complicated by hemorrhage, bowel obstruction, and perforation. ${ }^{11,12}$ If no other signs of bowel compromise are present, supportive care may be considered. The obstructive ileus in our patient was likely caused by the recurrent ulceration of PC, and spontaneously improved after the colonoscopy examination. The exact reason is unclear, but we believe that it may have been due to dislodgement of a fecalith, or that the scope passage attempt during the colonoscopy procedure may have caused some dilatation at the stricture site, which caused improvement of ileus. Since subsequent aggravation of colonic obstruction seemed probable, we recommended colectomy, but the patient refused. Spontaneous improvement of ileus in a patient with PC has previously been reported. ${ }^{6}$ Our patient also showed a self-limiting course after conservative care, but it is not clear whether a watchful waiting for future patients with PC should be mandatory.

In conclusion, we report a case of PC with a transverse colonic stricture in a patient with chronic kidney disease who had been taking multiple kinds of herbal medication for more than 6 months. In this patient, PC was improved by supportive care. Since PC is frequently associated with chronic kidney disease and contrast-enhanced CT is not feasible, early endoscopy seems to be required for early diagnosis of PC, especially if there are multiple calcifications found on the bowel wall. Further clinical experience is required to establish optimal treatment plans and to elucidate the prognosis of PC.

\section{REFERENCES}

1. Iwashita A, Yao T, Schlemper RJ, et al. Mesenteric phlebosclerosis: a new disease entity causing ischemic colitis. Dis Colon Rectum 2003;46:209-220.

2. Chang KM. New histologic findings in idiopathic mesenteric phlebosclerosis: clues to its pathogenesis and etiology--probably ingested toxic agent-related. J Chin Med Assoc 2007;70:227235.

3. Song JH, Kim JI, Jung JH, et al. A case of phlebosclerotic colitis in a hemodialysis patient. Korean J Gastroenterol 2012;59:40-43.

4. Kang HY, Noh R, Kim SM, Shin HD, Yun SY, Song IH. Phlebosclerotic colitis in a cirrhotic patient with portal hypertension: the first case in Korea. J Korean Med Sci 2009;24:1195-1199.

5. Yao T, Iwashita A, Hoashi T, et al. Phlebosclerotic colitis: value of radiography in diagnosis--report of three cases. Radiology 2000;214:188-192.

6. Hu P, Deng L. Phlebosclerotic colitis: three cases and literature review. Abdom Imaging 2013;38:1220-1224.

7. Markos V, Kelly S, Yee WC, Davis JE, Cheifetz RE, Alsheikh A. Phlebosclerotic colitis: imaging findings of a rare entity. AJR Am J Roentgenol 2005;184:1584-1586.

8. Hiramatsu K, Sakata H, Horita Y, et al. Mesenteric phlebosclerosis associated with long-term oral intake of geniposide, an ingredient of herbal medicine. Aliment Pharmacol Ther 2012;36:575-586.

9. Choi JM, Lee KN, Kim HS, et al. Idiopathic phlebosclerotic colitis: a rare entity of chronic ischemic colitis. Korean J Gastroenterol 2014;63:183-186.

10. Hirasaki S, Matsumura K. Development of phlebosclerotic colitis under treatment with Chinese herbal therapy. Intern Med 2014;53:1709-1710. 
11. Chang YY, Lin HH, Lin CC. Phlebosclerotic colitis presenting as intestinal obstruction. Clin Gastroenterol Hepatol 2014;12:e81e82. doi: 10.1016/j.cgh.2014.02.018.
12. Kato T, Miyazaki K, Nakamura T, Tan KY, Chiba T, Konishi F. Perforated phlebosclerotic colitis: description of a case and review of this condition. Colorectal Dis 2010;12:149-151. 